B.Y. CHEN

KODAI MATH. J.

8 (1985), 358-374

\title{
FINITE TYPE SUBMANIFOLDS IN PSEUDO-EUCLIDEAN SPACES AND APPLICATIONS
}

\author{
By BANG-YEN CHEN
}

\section{$\S 1$. Introduction.}

Let $E_{s}{ }^{m}$ be the $m$-dimensional pseudo-Euclidean space with (flat) pseudoRiemannian metric of signature $(s, m-s)$. And let $M$ be a compact space-like submanifold of $E_{s}{ }^{m}$. By using the induced Riemannian structure on $M$, we can define two well-defined numbers $p$ and $q$ associated with the submanifold $M$ in $E_{s}{ }^{m}$. Here $p$ is a positive integer and $q$ is either $+\infty$ or an integer $\geqq p$. The pair $[p, q]$ is called the order of the submanifold $M$ (cf. [1]). The submanifold $M$ is said to be of finite type if $q$ is finite. Otherwise, $M$ is said to be of infinte type. The submanifold $M$ is of finite type if and only if there is a non-trivial polynomial $P$ such $P(\Delta) H=0$; where $\Delta$ is the Laplacian $\Delta$ on $M$ and $H$ the mean curvature vector of $M$ in $E_{s}{ }^{m}$.

In this paper, we will give some general results for finite type submanifolds in the pseudo-Euclidean space $E_{s}{ }^{m}$. By applying these results, we will prove the following. (1) There exist no compact space-like hypersurfaces with constant mean curvature and constant scalar curvature in the anti-de Sitter space-time; (2) Every compact hypersurface with constant mean curvature and constant scalar curvature in a hyperbolic space is a small hypersphere; and (3) If $M$ is a compact space-like hypersurface of the de Sitter space-time, then $M$ has nonzero constant mean curvature and constant scalar curvature when and only when $M$ is mass-symmetric and of 2-type in the Lorentz-Minkowski world.

For the general knowledge on Finite-Type Submanifolds in Euclidean spaces, see $[1,2]$. And for the general knowledge on Relativity, see for instance $[3,4]$.

\section{$\S 2$. Preliminaries.}

Let $E_{s}{ }^{m}$ be the $m$-dimensional pseudo-Euclidean space with metric tensor given by

$$
g_{0}=-\sum_{i=1}^{s} d x_{i}^{2}+\sum_{\jmath=s+1}^{m} d x_{\jmath}^{2},
$$

where $\left(x_{1}, \cdots, x_{m}\right)$ is a rectangular coordinate system of $E_{s}{ }^{m} . \quad\left(E_{s}{ }^{m}, g_{0}\right)$ is a flat pseudo-Riemannian manifold of signature $(s, m-s)$.

Received February 5, 1985 
Let $c$ be a point in $E_{s}{ }^{m}$ and $r>0$. We put

$$
\begin{aligned}
& S_{s}{ }^{m}(c, r)=\left\{x \in E_{s}{ }^{m+1} \mid\langle x-c, x-c\rangle=r^{2}\right\}, \\
& H_{s}{ }^{m}(c, r)=\left\{x \in E_{s+1}{ }^{m+1} \mid\langle x-c, x-c\rangle=-r^{2}\right\},
\end{aligned}
$$

where $\langle$,$\rangle denotes the indefinite inner product on the pseudo-Euclidean space.$ It is known that $S_{s}{ }^{m}(c, r)$ and $H_{s}{ }^{m}(c, r)$ are complete pseudo-Riemannian manifolds of signature $(s, m-s)$ and respective constant sectional curvatures $r^{-2}$ and $-r^{-2}$. $\quad S_{s}{ }^{m}(c, r)$ is simply-connected for $s<m-1 ; S_{m-1}{ }^{m}(c, r)$ is connected and has infinite cyclic fundamental group; and $S_{m}{ }^{m}(c, r)$ has two simply-connected components. $S_{s}{ }^{m}(c, r)$ and $H_{s}{ }^{m}(c, r)$ are called the pseudo-Riemannian sphere and the pseudo-hyperbolic space, respectively (cf. [6, p. 67].) The point $c$ is called the center of $S_{s}{ }^{m}(c, r)$ and of $H_{s}{ }^{m}(c, r)$. In the following, $S_{s}{ }^{m}(0,1)$ and $H_{s}{ }^{m}(0,1)$ are simply denoted by $S_{s}{ }^{m}$ and $H_{s}{ }^{m}$, respectively. $S_{1}{ }^{m}$ is called the de Sitter space-tıme (=de Sitter world) and $H_{1}{ }^{m}$ the antı-de Sitter space-tıme (=antı-de Sitter world). Both $S_{1}{ }^{m}$ and $H_{1}{ }^{m}$ are pseudo-Riemannian manifold of signature $(1, m-1)$. The hyperbolic space $H^{m}$ is defined by

$$
H^{m}=\left\{x \in E_{1}{ }^{m+1} \mid\langle x, x\rangle=-1 \text { and } t>0\right\},
$$

where $t=x_{1}$ is the first coordinate in $E_{1}{ }^{m} . H^{m}$ is a complete, simply-connected Riemannian manifold of constant sectional curvature $-1 . E_{1}{ }^{m}$ is called the Lorentz-Minkowskı space-tıme (=Lorentz-Minkowskı world.)

Let $\tilde{M}$ be a pseudo-Riemannian manifold with pseudo-Riemannian metric $\tilde{g}$. Denote by $\langle$,$\rangle the associated non-degenerate inner product and by \tilde{\nabla}$ the metric connection on $\tilde{M}$. A tangent vector $X$ to $\tilde{M}$ is said to be space-like (respectively, time-like or light-like) if $\langle X, X\rangle>0$ or $X=0$ (respectively, if $\langle X, X\rangle\langle 0$ or $\langle X, X\rangle=0$ and $X \neq 0$ ).

Let $M$ be a submanifold of $\tilde{M}$. If the pseudo-Riemannian metric tensor $\tilde{g}$ of $\tilde{M}$ induces a pseudo-Riemannian metric (respectively, Riemannian metric) on $M$, then $M$ is called a psuedo-Riemannian (respectively, space-like) submanifold of $\tilde{M}$.

If $M$ is a pseudo-Riemannian (or space-like) submanifold of $\tilde{M}$, each tangent space is, $T_{x}(M)$ by definition, a nondegenerate subspace of $T_{x}(\tilde{M})$. Hence, we have the direct sum decomposition:

$$
T_{x}(\tilde{M})=T_{x}(M) \oplus T_{x}^{\perp}(M),
$$

where the normal space $T_{x}^{\perp}(M)$ is also nondegenerate.

Let $\nabla$ denote the induced metric connection on $M$. Then, for any vector fields $X, Y$ tangent to $M$, we have the following Gauss formula:

$$
\tilde{\nabla}_{X} Y=\nabla_{X} Y+h(X, Y),
$$

where $h$ is the second fundamental form of $M$ in $\tilde{M}$.

Denote by $R$ and $\widetilde{R}$ the curvature tensors of $M$ and $\tilde{M}$, respectively. The 
Gauss equation is given by

$$
\begin{aligned}
& \langle R(X, Y) Z, W\rangle=\langle\tilde{R}(X, Y) Z, W\rangle \\
& \quad+\langle h(X, W), h(Y, Z)\rangle-\langle h(X, Z), h(Y, W)\rangle .
\end{aligned}
$$

Denote by $D$ the linear connection induced on the normal bundle $T^{\perp}(M)$. For each vector field $\xi$ normal to $M$, the Weingarten formula is given by

$$
\tilde{\nabla}_{X} \xi=-A_{\xi} X+D_{X} \xi
$$

where $A_{\xi}$ is the Weingarten map with respect to $\xi$. $A_{\xi}$ is a self-adjoint endomorphism of the tangent bundle $T(M)$ which can be diagonalized when $M$ is space-like. It is well-known that $h$ and $A$ are related by

$$
\langle h(X, Y), \xi\rangle=\left\langle A_{\xi} X, Y\right\rangle \text {. }
$$

For the second fundamental form $h$, we define the covariant differentiation of $h$ by

$$
\left(\bar{\nabla}_{X} h\right)(Y, Z)=D_{X} h(Y, Z)-h\left(\nabla_{X} Y, Z\right)-h\left(Y, \nabla_{X} Z\right) .
$$

The Codazzi equation is given by

$$
(\tilde{R}(X, Y) Z)^{\perp}=\left(\bar{\nabla}_{X} h\right)(Y, Z)-\left(\bar{\nabla}_{Y} h\right)(X, Z) .
$$

A normal vector field $\xi$ is said to be parallel is $D_{X} \xi=0$ for any vector $X$ tangent to $M$.

Let $M$ be a pseudo-Riemannian submanifold with signature $(t, n-t)$ in $M$. Let $E_{1}, \cdots, E_{n}$ be an orthonormal local basis on $M$ such that $E_{1}, \cdots, E_{t}$ are time-like and $E_{t+1}, \cdots, E_{n}$ are space-like. If $F$ is an endomorphism of $T M$ such that $F=\left(F_{\imath j}\right)$ with respect to the basis $E_{1}, \cdots, E_{n}$, then the trace of $F$ is defined by

$$
\operatorname{tr} F=\sum_{\imath=1}^{n} \varepsilon_{i} F_{\imath \imath}
$$

where $\varepsilon_{1}=\cdots=\varepsilon_{t}=-1, \varepsilon_{t+1}=\cdots=\varepsilon_{n}=1$ and $F_{\imath \jmath}=\left\langle F\left(E_{\imath}\right), E_{\jmath}\right\rangle$. From these, we find

$$
\operatorname{tr} h=\sum_{\imath=1}^{n} \varepsilon_{i} h\left(E_{\imath}, E_{\imath}\right) .
$$

The mean curvature vector $H$ of $M$ in $\tilde{M}$ is defined by $H=1 / n \operatorname{tr} h$, where $n=\operatorname{dim} M$. A pseudo-Riemannian submanifold $M$ of $\tilde{M}$ is said to be minimal if the mean curvature vector $H$ vanishes identically. A pseudo-Riemannian submanifold $M$ in $\tilde{M}$ is said to be pseudo-umbilical if $\langle H, H\rangle \neq 0$ and $A_{H}=\mu I$ for some function $\mu$ on $M$, where $I$ is the identity transformation.

We need the following.

LEMMA 1. Let $M$ be a pseudo-Riemannian (in particular, space-like) submanifold of the pseudo-Riemannian sphere $S_{s}{ }^{m}(c, r)$ (respectively, the pseudo-hyperbolic 
space $\left.H_{s-1}{ }^{m}(c, r)\right)$ in $E_{s}{ }^{m+1}$. Then the mean curvature vectors $H$ and $H^{\prime}$ of $M$ in $E_{s}{ }^{m+1}$ and in $S_{s}{ }^{m}(c, r)$ (respectively, in $H_{s-1}{ }^{m}(c, r)$ ) satisfy

$$
H=H^{\prime}-\frac{1}{r^{2}}(x-c) \quad\left(\text { respectively, } H=H^{\prime}+\frac{1}{r^{2}}-(x-c)\right) \text {. }
$$

Proof. Let $\varepsilon=1$ or -1 according to $M$ being a submanifold of $S_{s}{ }^{m}(c, r)$ or of $H_{s-1}{ }^{m}(c, r)$. Let $x$ denote the position vector of $M$ in $E_{s}{ }^{m+1}$. Then we have

$$
\langle x-c, x-c\rangle=\varepsilon r^{2} .
$$

For each vector $X$ tangent to $S_{s}{ }^{m}(c, r)$ or to $H_{s-1}{ }^{m}(c, r)$ we have $\tilde{\nabla}_{X} x=X$. Thus, $x-c$ is normal to $S_{s}{ }^{m}(c, r)$ and to $H_{s-1}{ }^{m}(c, r)$. Moreover, from $\dot{\nabla}_{X} x=X$, we have

$$
A_{x-c}=\tilde{A}_{x-c}=-I \text {, }
$$

where $A$ and $\tilde{A}$ denote the Weingarten maps of $M$ and $S_{s}{ }^{m}(c, r)$ (or $H_{s-1}{ }^{m}(c, r)$ ) in $E_{s}{ }^{m+1}$. Let $h^{\prime}$ and $h$ be the second fundamental forms of $M$ in $S_{s}{ }^{m}(c, r)$ (or in $\left.H_{s-1}{ }^{m}(c, r)\right)$ and of $S_{s}{ }^{m}(c, r)$ (or $\left.H_{s-1}{ }^{m}(c, r)\right)$ in $E_{s}{ }^{m+1}$, respectively. Then we have

$$
h(X, Y)=h^{\prime}(X, Y)+\tilde{h}(X, Y) .
$$

Since $h(X, Y)=\varepsilon\left\langle A_{x-c} X, Y\right\rangle(x-c) / r^{2},(2.12),(2.15)$ and (2.16) give (2.13).

(Q.E. D.)

\section{$\S 3$. Some General Results.}

Throughout the remaining part of this paper, we assume that $M$ is a connected, $n$-dimensional, pseudo-Riemannian submanifold of $E_{s}{ }^{m+1}$.

First, we give the following.

Lemma 2. A submanifold $M$ of the pseudo-Euclidean space $E_{s}{ }^{m+1}$ is a pseudoumbilical submanifold with parallel mean curvature vector if and only if $M$ is either a minimal submanifold of a pseudo-Riemannian sphere $S_{s}{ }^{m}(c, r)$ or a minimal submanifold of a psedo-hyperbolic space $H_{s-1}{ }^{m}(c, r)$ for some $c \in E_{s}{ }^{m+1}$ and $r>0$.

Proof. Assume that $M$ is a pseudo-umbilical submanifold of $E_{s}{ }^{m+1}$ with parallel mean curvature vector. Then, $\langle H, H\rangle \neq 0$ and, by definition, for any $X$ tangent to $M$, we have $X\langle H, H\rangle=2\left\langle\tilde{\nabla}_{X} H, H\right\rangle=0$. Thus, $\langle H, H\rangle$ is a non-zero constant. We put

$$
\langle H, H\rangle=\frac{\varepsilon}{r^{2}}, \quad \varepsilon=1 \text { or }-1 .
$$

Let $A_{H}=\mu I$. Then from (2.9) and (2.12), we find

$$
\varepsilon=\mu r^{2} \text {. }
$$

We put 


$$
y=x+\varepsilon r^{2} H,
$$

where $x$ is the position vector of $M$ in $E_{s}{ }^{m+1}$. Then, for any vector $X$ tangent to $M$, we have

$$
\begin{aligned}
\tilde{\nabla}_{X} y & =\tilde{\nabla}_{X} x+\varepsilon r^{2} \tilde{\nabla}_{X} H \\
& =X-\varepsilon r^{2} A_{H} X=0 .
\end{aligned}
$$

Thus $y$ is a constant vector, say $c$, in $E_{s}{ }^{m+1}$. Hence, we find $\langle x-c, x-c\rangle=$ $r^{4}\langle H, H\rangle=\varepsilon r^{2}$. This shows that $M$ lies either in the pseudo-Riemannian sphere $S_{s}{ }^{m}(c, r)$ or in the pseudo-hyperbolic space $H_{s-1}{ }^{m}(c, r)$. Since (3.3) gives

$$
H=-\frac{\varepsilon}{r^{2}}(x-c),
$$

Lemma 1 implies that $H^{\prime}=0$, i. e., $M$ is a minimal submanifold of $S_{s}{ }^{m}(c, r)$ or of $H_{s-1}{ }^{m}(c, r)$.

Conversely, if $M$ is a minimal submanifold of $S_{s}{ }^{m}(c, r)$ or of $H_{s-1}{ }^{m}(c, r)$, then Lemma 1 shows that $H=-\varepsilon(x-c) / r^{2}$. This implies that

$$
\tilde{\nabla}_{X} H=-\frac{\varepsilon}{r^{2}} \tilde{\nabla}_{X} x=-\frac{\varepsilon}{r^{2}} X .
$$

Thus, $A_{H}=\varepsilon I / r^{2}$ and $D H=0$. Moreover, by applying (2.13) and (3.5) we also find $\langle H, H\rangle=\varepsilon / r^{2} \neq 0$. Consequently, $M$ is a pseudo-umbilical submanifold with parallel mean curvature vector.

(Q.E. D.)

Remark 1. If $E_{s}{ }^{m+1}$ is the Euclidean space $E^{m+1}$, Lemma 2 is due to Yano and Chen [7]. For pseudo-Euclidean case the "only if" part of Lemma 2 was given in [5].

LEMMA 3. If $M$ is a submanfold of $S_{s}{ }^{m}$ (or $H_{s-1}{ }^{m}$ ) in $E_{s}{ }^{m+1}$, then the mean curvature vectors $H^{\prime}$ and $H$ in $S_{s}{ }^{m}$ (or in ${H_{s-1}}^{m}$ ) and in $E_{s}{ }^{m+1}$ satisfy

$$
D H=D^{\prime} H^{\prime}, \quad A_{H}=A_{H H^{\prime}}+\varepsilon I .
$$

Proof. Obvious from (2.8), (2.14) and Lemma 1.

In the following, by a hyperplane section $N$ of $S_{s}{ }^{m}$ (or of $H_{s-1}{ }^{m}$ ) we mean the intersection of $S_{s}{ }^{m}$ (or of $H_{s-1}{ }^{m}$ ) and a hyperplane $L$ of $E_{s}{ }^{m+1}$.

By applying Lemma 2, we have the following.

Proposition 1. Let $M$ be a submanifold of the pseudo-Riemannian sphere $S_{s}{ }^{m}$ (respectively, of the pseudo-hyperbolic space $H_{s-1}{ }^{m}$.) If $M$ is a pseudo-umbilical submanifold with parallel mean curvature vector, then $M$ is a minimal submanifold of a hyperplane section of $S_{s}{ }^{m}$ (respectively, of ${H_{s-1}}^{m}$ ).

Proof. Under the hypothesis, the mean curvature vector $H^{\prime}$ of $M$ in $S_{s}{ }^{m}$ (or in $H_{s-1}{ }^{m}$ ) satisfies

$$
\left\langle H^{\prime}, H^{\prime}\right\rangle \neq 0, \quad A_{H^{\prime}}^{\prime}=\mu I \text { and } \quad D^{\prime} H^{\prime}=0,
$$


where $A^{\prime}$ and $D^{\prime}$ denote the Weingarten map and the normal connection of $M$ in $S_{s}{ }^{m}$ (or in $H_{s-1}{ }^{m}$ ). Let $A$ be the Weingarten map of $M$ in $E_{s}{ }^{m+1}$. Then, by (3.7) and Lemma 3, we know that $M$ has parallel mean curvature vector $H$ in $E_{s}{ }^{m+1}$, too. Since $H$ is parallel, $\langle H, H\rangle$ is constant.

If $\langle H, H\rangle=0$, then $H=H^{\prime}-\varepsilon x$ is a light-like vector. Thus, we have $\left\langle H^{\prime}, H^{\prime}\right\rangle=-\varepsilon$. Because $A^{\prime}{ }_{H^{\prime}}=\mu I$, we find $\mu=\left\langle H^{\prime}, H^{\prime}\right\rangle=-\varepsilon$. Thus, we get

$$
A_{H^{\prime}}=A_{H^{\prime}}^{\prime}=-\varepsilon I \text {. }
$$

Applying (3.7), (3.8) and Lemma 3, we find

$$
\tilde{\nabla}_{X} H=\tilde{\nabla}_{X} H^{\prime}-\varepsilon \tilde{\nabla}_{X} x=0 .
$$

This shows that $H$ is a constant vector in $E_{s}{ }^{m+1}$. Let $H=-\varepsilon c$. Then we have

$$
\langle x-c, x-c\rangle=-\varepsilon .
$$

Since $\langle x, x\rangle=\varepsilon$, we obtain $\langle x, c\rangle=\varepsilon-1 / 2\langle c, c\rangle$. This shows that $M$ lies in the hyperplane section $N$ given by

$$
N=\left\{x \in E_{s}{ }^{m+1} \mid\langle x, x\rangle=\varepsilon \text { and }\langle x, c\rangle=\varepsilon-1 / 2\langle c, c\rangle\right\}
$$

for some constant $c$. Since $H=-\varepsilon c$ is normal to the hyperplane $\left\{x \in E_{s}{ }^{m+1} \mid\langle x, c\rangle\right.$ $=\varepsilon-1 / 2\langle c, c\rangle\}$, the mean curvature vector of $M$ in $N$ vanishes. Thus, $M$ is a minimal submanifold of $N$.

If $\langle H, H\rangle \neq 0$, then $M$ is pseudo-umbilical in $E_{s}{ }^{m+1}$ by Lemma 2. Thus, Lemma 2 implies that $M$ is a minimal submanifold of a $S_{s}{ }^{m}(c, r)$ or of a $H_{s-1}{ }^{m}(c, r)$ for some $c \in E_{s}{ }^{m+1}$ and $r>0$. Thus, we have

$$
\langle x-c, x-c\rangle=\bar{\varepsilon} r^{2},
$$

where $\bar{\varepsilon}=1$ or -1 according to $M$ is a minimal submanifold of $S_{s}{ }^{m}(c, r)$ or of $H_{s-1}{ }^{m}(c, r)$. Since $\langle x, x\rangle=\varepsilon$, (3.11) gives $2\langle x, c\rangle=\varepsilon-\bar{\varepsilon} r^{2}+\langle c, c\rangle$. Thus, $M$ lies in the hyperplane section given by

$$
N=\left\{x \in E_{s}{ }^{m+1} \mid\langle x, x\rangle=\varepsilon \text { and }\langle x-c, x-c\rangle=\bar{\varepsilon} r^{2}\right\} .
$$

Since $M$ is minimal in $\left\{x \in E_{s}{ }^{m+1} \mid\langle x-c, x-c\rangle=\bar{\varepsilon} r^{2}\right\}, M$ is minimal in $N$, too.

$$
\text { (Q.E. D.) }
$$

Remark 2. If $M$ is a minimal submanifold of a hyperplane section $N$ of $S_{s}{ }^{m}$ (or of $H_{s-1}{ }^{m}$ ), then either $N$ is totally geodesic in $S_{s}{ }^{m}$ (or in $H_{s-1}{ }^{m}$ ) or $N$ is a pseudo-umbilical submanifold with parallel mean curvature vector.

Let $M$ be a pseudo-Riemannian submanifold with orthonormal local basis $E_{1}, \cdots, E_{n}$. For any real function $f$ on $M$, the Laplacian $\Delta f$ of $f$ is defined by

$$
\Delta f=-\sum_{\imath=1}^{n} \varepsilon_{\imath}\left\{E_{\imath} E_{\imath} f-\nabla_{E_{i}} E_{\imath} f\right\} .
$$

We mention the following lemma for later use. 
Lemma 4. Let $M$ be an n-dimensional submanifold of $E_{s}{ }^{m+1}$. Then we have

$$
\Delta x=-n H \text {. }
$$

Proof. Let $a$ be any fixed vector in $E_{s}{ }^{m+1}$ and $p$ a point in $M$. Let $E_{1}, \cdots, E_{n}$ be an orthonormal local basis about $p$ such that $\left(\nabla_{E_{i}} E_{j}\right)(p)=0 ; i, j=$ $1, \cdots, n$. Then we have

$$
\begin{aligned}
(\Delta\langle x, a\rangle)_{p} & =-\sum_{\imath=1}^{n} \varepsilon_{i}\left(E_{\imath}\right)_{p}\left\langle E_{\imath}, a\right\rangle \\
& =-\sum \varepsilon_{i}\left\langle\tilde{\nabla}_{E_{i}} E_{\imath}, a\right\rangle(p) \\
& =-\sum \varepsilon_{i}\left\langle h\left(E_{\imath}, E_{\imath}\right), a\right\rangle_{p} \\
& =-n\langle H, a\rangle_{p} .
\end{aligned}
$$

Since both $\Delta x$ and $H$ are independent of the choice of the local basis, we have $\langle\Delta x, a\rangle=-\langle n H, a\rangle$. Because the inner product $\langle$,$\rangle is nondegenerate, this$ implies equation (3.12).

(Q.E.D.)

Combining Lemmas 1 and 4 we have the following.

PROPOSITION 2. There exist no compact space-like minimal submanifolds in any pseudo-hyperbolic space $H_{s}{ }^{m}$.

Proof. If $M$ is a compact space-like minimal submanifold of $H_{s}{ }^{m}$, then Lemma 1 gives $H=x$. Thus, Lemma 4 implies $\Delta x=-n x$. This shows that $-n$ is an eigenvalue of $M$. Since $M$ is a compact Riemannian manifold, eigenvalues of $\Delta$ on $M$ are non-negative.

(Q.E.D.)

Remark 3. In contrast to Proposition 2, there exist compact minimal spacelike submanifolds in pseudo-Riemannian spheres.

LEMMA 5. If $M$ is compact space-like submanifold of $E_{s}{ }^{m+1}$, then we have

$$
\begin{aligned}
& \int_{M} H d V=0, \\
& \int_{M}\langle H, x\rangle d V+\int_{M} d V=0 .
\end{aligned}
$$

Proof. Since $M$ is compact, Lemma 4 and Hopf's Lemma implies (3.13). (3.14).

By using (3.12) we have $\Delta\langle x, x\rangle=-2 n(1+\langle H, x\rangle)$. Thus, we also have

In the following, a compact submanifold $M$ of $S_{s}{ }^{m}(c, r)$ (or of $H_{s-1}{ }^{m}(c, r)$ ) in $E_{s}{ }^{m+1}$ is called mass-symmetric if the center of mass of $M$ in $E_{s}{ }^{m+1}$ is just the center $c$ of $S_{s}{ }^{m}(c, r)$ (or of $H_{s-1}{ }^{m}(c, r)$ ). Lemmas 1 and 4 imply the following.

LEMMA 6. If $M$ is a compact, space-like, minimal submanifold of the pseudoRiemannian sphere $S_{s}{ }^{m}$, then $M$ is mass-symmetric in $E_{s}{ }^{m+1}$. 
Proof. Under the hypothesis, we have $\Delta x=-n H=n x$. Thus $\int_{M} x d V=0$. (Q.E.D.)

Remark 4. From the proof of Lemma 6, we see that if $M$ is an $n$-dimensional, compact, space-like, minimal submanifold of $S_{s}{ }^{m}$, then the first non-zero eigenvalue $\lambda_{1}$ of $\Delta$ on $M$ satisfies $\lambda_{1} \leqq n$.

\section{$\S 4$. Finite-type submanifolds in $E_{s}{ }^{m+1}$.}

Let $M$ be a compact, space-like submanifold of $E_{s}{ }^{m+1}$. Then $M$ with the induced metric is a Riemannian manifold. Thus, the Laplacian $\Delta$ of $M$ is an elliptic differential operator and it has infinite sequence of eigenvalues:

$$
0=\lambda_{0}<\lambda_{1}<\lambda_{2}<\cdots<\lambda_{k}<\cdots \uparrow \infty .
$$

Let $V_{k}=\left\{f \in C^{\infty}(M) \mid \Delta f=\lambda_{k} f\right\}$ be the eigenspace of $\Delta$ with eigenvalue $\lambda_{k}$. Then each $V_{k}$ is finite-dimensional. If we define an inner product on $C^{\infty}(M)$ by $(f, g)=\int_{M} f g d V$, then the decomposition $\sum_{k=0}^{\infty} V_{k}$ is orthogonal and dense in $C^{\infty}(M)$ (in $L^{2}$-sense). $V_{0}$ is 1-dimensional and it consists of constant functions.

For each $f \in C^{\infty}(M)$, let $f_{t}$ be the projection of $f$ onto $V_{t}$. Then we have the following decomposition:

$$
f=\sum_{t=0}^{\infty} f_{t} \quad\left(\text { in } L^{2} \text {-sense }\right) .
$$

If $f$ is a non-constant function on $M$, there is a positive integer $p \geqq 1$ such that $f_{p} \neq 0$ and $f=f_{0}+\sum_{t \geq p} f_{t}$. If there are infinite $f_{t}$ 's which are nonzero, we put $q=\infty$. Otherwise, there is an integer $q \geqq p$ such that $f_{q} \neq 0$ and $f=f_{0}+\sum_{t=p}^{q} f_{t}$. Thus, in both cases, we have

$$
f-f_{0}=\sum_{t=p}^{q} f_{t}
$$

where $q$ is either $\infty$ or an integer $\geqq p$.

For the compact, space-like submanifold $M$ in $E_{s}{ }^{m+1}$, we put

$$
x=\left(x_{1}, \cdots, x_{m+1}\right),
$$

where $x_{A}$ is the $A$-th rectangular coordinate function of $M$ in $E_{s}{ }^{m+1}$. For each $x_{A}$, we have

$$
x_{A}-\left(x_{A}\right)_{0}=\sum_{t=p_{A}}^{q_{A}}\left(x_{A}\right)_{t}, \quad A=1, \cdots, m+1
$$

We put

$$
p=\inf \left\{p_{A}\right\}, \quad q=\sup \left\{q_{A}\right\},
$$

where $A$ ranges among all $A$ such that $x_{A} \neq\left(x_{A}\right)_{0}$. It is clear that $p$ is an 
integer $\geqq 1$ and $q$ is either $\infty$ or an integer $\geqq p$. The pair of these invariants $[p, q]$ is called the order of $M$ in $E_{s}{ }^{m+1}$ (cf. [1]). The submanifold $M$ in $E_{s}{ }^{m+1}$ is said to be of finite type if $q$ is finite. Otherwise, $M$ is of infinite type. The submanifold $M$ in $E_{s}{ }^{m+1}$ is said to be of $k$-type if there exist exactly $k$ nonzero $x_{t}$ 's in the following decomposition:

$$
x=x_{0}+\sum_{t=p}^{q} x_{t}, \quad \Delta x_{t}=\lambda_{t} x_{t} .
$$

We need the following.

Proposition 3. Let $M$ be a compact, space-like submanifold of $E_{s}{ }^{m+1}$. Then $M$ is of fintte type if and only if there exists a non-trivial polynomial $P$ such that $P(\Delta) H=0$ (or equivalently, $P(\Delta)\left(x-x_{0}\right)=0$ ).

Proof. Consider the decomposition (4.7). If $M$ is of finite type, $q$ is finite. Thus, we have from Lemma 4 ,

$$
-n \Delta^{2} H=\sum_{t=p}^{q} \lambda_{t}^{2+1} x_{t}, \quad i=0,1,2, \cdots .
$$

Let $c_{1}=-\sum_{t=p}^{q} \lambda_{t}, c_{2}=\sum_{t<s} \lambda_{t} \lambda_{s}, \cdots, c_{q-p+1}=(-1)^{q-p+1} \lambda_{p} \cdots \lambda_{q}$. Then we find

$$
\Delta^{k} H+c_{1} \Delta^{k-1} H+\cdots+c_{k} H=0, \quad k=q-p+1 .
$$

Conversely, if $H$ satisfies (4.9) for some $k \geqq 0$, then $k \geqq 1$ by Lemma 4 . Thus, by (4.7) and (4.9), we find

$$
\sum_{t=1}^{\infty} \lambda_{t}\left(\lambda_{t}^{k}+c_{1} \lambda_{t}^{k-1}+\cdots+c_{k}\right) x_{t}=0 .
$$

Let

Then (4.10) gives

$$
x_{t}=\left(\left(x_{t}\right)_{1}, \cdots,\left(x_{t}\right)_{m+1}\right) .
$$

$$
\sum_{t=1}^{\infty} \lambda_{t}\left(\lambda_{t}{ }^{k}+c_{1} \lambda_{t}{ }^{k-1}+\cdots+c_{k}\right)\left(x_{t}\right)_{A}=0, \quad A=1, \cdots, m+1 .
$$

If $x_{s} \neq 0$, then

for some $A$. Thus, we find

$$
\left(x_{s}\right)_{A} \neq 0
$$

$$
0=\sum_{t=1}^{\infty} \lambda_{t}\left(\lambda_{t}{ }^{k}+c_{1} \lambda_{t}{ }^{k-1}+\cdots+c_{k}\right)\left(\left(x_{t}\right)_{A},\left(x_{s}\right)_{A}\right)=0,
$$

where $(f, g)=\int_{M} f g d V$. Since

we find

$$
\begin{aligned}
& \lambda_{t}\left(\left(x_{t}\right)_{A},\left(x_{s}\right)_{A}\right)=\left(\Delta\left(x_{t}\right)_{A},\left(x_{s}\right)_{A}\right) \\
& \quad=\left(\left(x_{t}\right)_{A}, \Delta\left(x_{s}\right)_{B}\right)=\lambda_{s}\left(\left(x_{t}\right)_{A},\left(x_{s}\right)_{A}\right),
\end{aligned}
$$




$$
\left(\left(x_{t}\right)_{A},\left(x_{s}\right)_{A}\right)=0
$$

if $t \neq s$. Therefore, (4.12) implies

$$
\lambda_{t}{ }^{k}+c_{1} \lambda_{t}{ }^{k-1}+\cdots+c_{k}=0 \quad \text { whenever } \quad x_{t} \neq 0 .
$$

Since equation (4.13) has at most $k$ real solutions, at most $k$ of the $x_{t}$ 's in (4.7) are nonzero. Thus, $M$ is of finite-type. Similar argument applies to $P(\Delta)\left(x-x_{0}\right)$ $=0$.

From the proof of Proposition 3, we also have the following.

(Q.E.D)

Proposition 4. Let $M$ be a compact, space-like submanifold of $E_{s}{ }^{m+1}$. If $M$ is of $k$-type, then there exists a polynomial $P$ of degree $k$ such that $P(\Delta) H=0$ (or $P(\Delta)\left(x-x_{0}\right)=0$.) Conversely, if $P(\Delta) H=0$ (or $\left.P(\Delta)\left(x-x_{0}\right)=0\right)$ for a polynomal $P$ of degree $d \geqq 1$, then $M$ is of $k$-type for some $k ; d \geqq k \geqq 1$.

Now, we give the following Lemmas for later use.

LEMMA 7. Let $M$ be a compact, space-like submanifold of $E_{s}{ }^{m+1}$. If $M$ is not of 1-type and if $H$ satisfies $\Delta^{2} H+b \Delta H+c H=0$ for some constant $b$ and $c$, then $b=-\left(\lambda_{p}+\lambda_{q}\right)<0$ and $c=\lambda_{p} \lambda_{q}>0$ where $[p, q]$ is the order of $M$ in $E_{s}{ }^{m+1}$.

Proof. Under the hypothesis, Proposition 4 shows that $M$ is of 2-type. Thus, we have $x=x_{0}+x_{p}+x_{q}$. Therefore, we find

$$
\left(\lambda_{p}{ }^{3}+b \lambda_{p}{ }^{2}+c \lambda_{p}\right) x_{p}+\left(\lambda_{q}{ }^{3}+b \lambda_{q}{ }^{2}+c \lambda_{q}\right) x_{q}=0 .
$$

Thus, we have $\lambda_{p}{ }^{2}+b \lambda_{p}+c=0, \lambda_{q}{ }^{2}+b \lambda_{q}+c=0$. Since $\lambda_{p}$ and $\lambda_{q}$ are positive real roots of $t^{2}+b t+c=0$, we have $b=-\left(\lambda_{p}+\lambda_{q}\right)<0$ and $c=-\lambda_{p} \lambda_{q}>0$.

(Q.E. D)

LEMMA 8. Let $M$ be a compact, space-like submanifold of $E_{s}{ }^{m+1}$. Then $x_{0}$ is the center of mass in $E_{s}{ }^{m+1}$.

Proof. Since $\int_{M} x_{t} d V=1 / \lambda_{t} \int_{M} \Delta x_{t} d V=0$, (4.9) gives to $x_{0}=\int_{M} x d V / \int_{M} d V$.

Lemma 9. Let $M$ be a space-like submanfold of $E_{s}{ }^{m+1}$. Then we have

$$
\Delta H=\Delta^{D} H+\sum_{\imath=1}^{n}\left\{\left(\nabla_{E_{i}} A_{H}\right) E_{i}+A_{D_{E_{i}}{ }_{H}} E_{i}+h\left(E_{\imath}, A_{H} E_{\imath}\right)\right\},
$$

where $\Delta^{D}$ is the Laplacian associated with $D$ and $E_{1}, \cdots, E_{n}$ an orthonormal local basis of $M$.

Proof. Let $a$ be any vector in $E_{s}{ }^{m+1}$ and $X, Y$ any vector fields tangent to M. We have

$$
\begin{aligned}
Y X\langle H, a\rangle= & \left\langle D_{Y} D_{X} H, a\right\rangle-\left\langle\nabla_{Y}\left(A_{H} X\right), a\right\rangle \\
& -\left\langle A_{D_{X} H} Y, a\right\rangle-\left\langle h\left(Y, A_{H} X\right), a\right\rangle .
\end{aligned}
$$


Since $\langle$,$\rangle is non-degenerate, this gives (4.15) where$

$$
\Delta^{D} H=-\sum_{i=1}^{n}\left\{D_{E_{i}} D_{E_{i}} H-D_{\nabla_{E_{i}} E_{i}} H\right\}
$$

\section{$\S 5$. 1-type Submanifolds in $E_{s}{ }^{m+1}$.}

In this section, we give the following characterization theorem for 1-type submanifolds.

Theorem 1. Let $M$ be a compact, space-like submanifold of $E_{s}{ }^{m+1}$. Then $M$ is of 1-type in $E_{s}{ }^{m+1}$ if and only if $M$ is a minimal submanifold in a pseudoRiemannian sphere $S_{s}{ }^{m}(c, r)$ for some $c \in E_{s}{ }^{m+1}$ and $r>0$.

Proof. If $M$ is of 1-type in $E_{s}{ }^{m+1}$, we have $\Delta x=\lambda_{p}\left(x-x_{0}\right)$. Thus, by Lemma 4 , we find

$$
n H=\lambda_{p}\left(x_{0}-x\right) .
$$

This shows that $n \tilde{\nabla}_{X} H=-\lambda_{p} X$. Thus, we have

$$
A_{H}=\frac{\lambda_{p}}{n} I \text { and } D H=0 \text {. }
$$

Moreover, by (5.1), we also have

$$
X\left\langle x-x_{0}, x-x_{0}\right\rangle=2\left\langle X, x-x_{0}\right\rangle=0
$$

for any $X$ tangent to $M$. Thus, $\left\langle x-x_{0}, x-x_{0}\right\rangle$ is constant.

If $\left\langle x-x_{0}, x-x_{0}\right\rangle=0$, then $M$ lies in the light cone $C=\left\{x \in E_{s}{ }^{m+1} \mid\left\langle x-x_{0}\right.\right.$, $\left.\left.x-x_{0}\right\rangle=0\right\}$ with vertex at $x_{0}$. Since $M$ lies in $C$ and $x_{0}$ is the center of mass of $M$ in $E_{s}{ }^{m+1}, M$ must lies in both parts of the light cone $C$. This is impossible since $M$ is assumed to be a space-like submanifold. Thus, $\langle H, H\rangle \neq 0$. Consequently, $M$ is pseudo-umbilical in $E_{s}{ }^{m+1}$ with parallel mean curvature vector. Thus, by Lemma $2, M$ is either a minimal submanifold of a pseudoRiemannian sphere or a minimal submanifold of a pseudo-hyperbolic space. Since $M$ is compact, Proposition 2 shows that the second case cannot occurs.

Conversely, if $M$ is a minimal submanifold of a pseudo-Riemannian sphere, then Lemmas 1 and 4 imply that $\Delta H+\lambda H=0$ for some constant $\lambda$. Thus, by Proposition 4, $M$ is of 1-type in $E_{s}{ }^{m+1}$.

(Q.E.D.)

In the following, a compact hypersurface $N$ of the pseudo-hyperbolic space $H_{s-1}{ }^{m}$ is called a small hypersphere of ${H_{s-1}}^{m}$ if $N$ is the intersection of $H_{s-1}{ }^{m}$ with a pseudo-Riemannian sphere $S_{s}{ }^{m}(c, r)$ in $E_{s}{ }^{m+1}$. A small hypersphere of $H_{s-1}{ }^{m}$ is a totally umbilical submanifold in both $H_{s-1}{ }^{m}$ and $E_{s}{ }^{m+1}$. Moreover, a small hypersphere of $H_{s-1}{ }^{m}$ is a totally umbilical hypersurface of a linear hyperplane $L$ in $E_{s}{ }^{m+1}$ with $c$ as its hyperplane normal.

By applying Theorem 1, we may obtain the following.

COROLlARY 1. Let $M$ be a compact, space-like submanifold of a pseudo- 
hyperbolic space $H_{s-1}{ }^{m}$ in $E_{s}{ }^{m+1}$. Then $M$ is of 1-type in $E_{s}{ }^{m+1}$ if and only if $M$ is a minimal submanifold of a small hypersphere of $H_{s-1}{ }^{m}$.

In particular, if $E_{s}{ }^{m-1}$ is the Lorentz-Minkowski space-time, Corollary 1 reduces to the following.

CoRollary 2. A compact submanifold $M$ of the hyperbolic space $H^{m} \subset E_{1}{ }^{m+1}$ is of 1-type in the Lorentz-Minkowski world if and only if $M$ is a minimal submanifold of a small hypersphere of $H^{m}$.

\section{§ 6. Hypersurfaces in Anti-de Sitter World.}

It is known that there exist abundant examples of compact hypersurfaces with constant mean curvature and constant scalar curvature in a Riemannian sphere $S^{m}$. In this section, by applying the theory of finite-type submanifolds, we prove the following non-existence theorem in the anti-de Sitter world.

THEOREM 2. There exist no compact space-like hypersurfaces with constant mean curvature and constant scalar curvature in the anti-de Sitter space-time $\mathrm{H}_{1}{ }^{n+1}$.

Proof. We regard the anti-de Sitter space-time $H_{1}{ }^{n+1}$ as a hypersurface of $E_{2}{ }^{n+2}$ defined by

$$
H_{1}{ }^{n+1}=\left\{x \in E_{2}{ }^{n+2} \mid\langle x, x\rangle=-1\right\}
$$

Let $M$ be a compact space-like hypersurface of $H_{1}{ }^{n+1}$. Then the position vector $x$ of $M$ in $E_{2}{ }^{n+2}$ is a time-like unit normal vector. From Lemma 1, we have

$$
H=H^{\prime}+x,
$$

where $H^{\prime}$ is the mean curvature vector of $M$ in $H_{1}{ }^{n+1}$. Since $M$ is space-like, $H^{\prime}$ is either zero or time-like. We put

$$
\left\langle H^{\prime}, H^{\prime}\right\rangle=-\alpha^{2} .
$$

Then $H^{\prime}=\alpha \xi$ for a unit time-like vector field $\xi$ normal to $M$ and tangent to $H_{1}{ }^{n+1}$. Moreover, we have $\operatorname{tr} A_{\xi}=-n \alpha$. By applying (2.9), we find

$$
\begin{aligned}
\sum_{\imath}\left\langle h\left(E_{\imath}, A_{H} E_{\imath}\right), x\right\rangle & =\sum_{\imath}\left\langle A_{H} E_{\imath}, A_{x} E_{\imath}\right\rangle=-\sum_{\imath}\left\langle A_{I I} E_{\imath}, E_{\imath}\right\rangle \\
& =-n\langle H, H\rangle=n+n \alpha^{2} .
\end{aligned}
$$

Similarly, applying (2.9) and (5.2) and Lemma 2, we also have

$$
\begin{aligned}
\sum_{\imath}\left\langle h\left(E_{\imath}, A_{H} E_{\imath}\right), \xi\right\rangle & =\alpha\left\|A_{\xi}\right\|^{2}-\operatorname{tr} A_{\xi} \\
& =\alpha\left\|A_{\hat{\xi}}\right\|^{2}+n \alpha .
\end{aligned}
$$

Consequently, (6.4) and (6.5) give 


$$
\sum_{\imath} h\left(E_{\imath}, A_{H} E_{\imath}\right)=-\left(\left\|A_{\xi}\right\|^{2}+n\right) H^{\prime}-\left(n+n \alpha^{2}\right) x .
$$

Since $A_{\xi}$ is self-adjoint and $M$ is space-like, we may diagonalize $A_{\xi}$. Let $E_{1}, \cdots, E_{n}$ be an orthonormal local basis of $M$ such that

$$
A_{\xi} E_{\imath}=\mu_{i} E_{\imath}, \quad \imath=1, \cdots, n .
$$

If we put

$$
\nabla_{E_{i}} E_{\jmath}=\sum_{k=1}^{n} \omega_{j}^{k}\left(E_{\jmath}\right) E_{k}
$$

then (6.7) implies

$$
\left(\nabla_{E_{\imath}} A_{H}\right) E_{\jmath}=\left(E_{i} \alpha\right) \mu_{j} E_{j}+\alpha\left(E_{i} \mu_{j}\right) E_{j}+\sum_{k} \alpha\left(\mu_{\jmath}-\mu_{k}\right) \omega_{\jmath}{ }^{k}\left(E_{\imath}\right) E_{k} .
$$

Therefore, by the Codazzi equation, we obtain

$$
\begin{array}{r}
\alpha\left\{\left(E_{i} \mu_{j}\right) E_{j}-\left(E_{\jmath} \mu_{\imath}\right) E_{i}+\sum_{k}\left(\mu_{j}-\mu_{k}\right) \omega_{j}{ }^{k}\left(E_{\imath}\right) E_{k}\right. \\
\left.-\sum_{k}\left(\mu_{\imath}-\mu_{k}\right) \omega_{i}{ }^{k}\left(E_{j}\right) E_{k}\right\}=0,
\end{array}
$$

where we have used the fact $D \xi=0$. If $\jmath \neq \imath,(6.9)$ gives

$$
\alpha\left(E_{i} \mu_{\jmath}\right)=\alpha\left(\mu_{i}-\mu_{\jmath}\right) \omega_{i}{ }^{\jmath}\left(E_{j}\right), \quad \jmath \neq \imath .
$$

Since $\omega_{i}{ }^{j}=-\omega_{j}{ }^{2},(6.8)$ and (6.10) imply

$$
\sum_{\imath}\left(\nabla_{E_{i}} A_{H}\right) E_{\imath}=\sum_{\imath}\left[\left(E_{\imath} \alpha\right) \mu_{i} E_{i}+\alpha\left(E_{i} \mu_{\imath}\right) E_{\imath}\right]+\alpha \sum_{j \neq \imath}\left(E_{j} \mu_{\imath}\right) E_{\jmath} .
$$

Since $\sum_{i} \mu_{\imath}=\operatorname{tr} A_{\xi}=-n \alpha$, (5.11) gives

$$
\sum_{\imath}\left(\nabla_{E_{i}} A_{H}\right) E_{\imath}=\sum_{\imath}\left[A_{D^{\prime}{ }_{i}{ }^{\prime}} E_{\imath}-\frac{n}{2}\left(E_{i} \alpha^{2}\right) E_{\imath}\right] \text {. }
$$

Consequently, by applying Lemma 2, Lemma 8, (6.6), (6.12), we find

$$
\begin{aligned}
\Delta H= & \Delta^{D^{\prime}} H^{\prime}+2 \operatorname{tr} A_{D^{\prime} H^{\prime}}+\frac{n}{2} \operatorname{grad}\left\langle H^{\prime}, H^{\prime}\right\rangle \\
& -\left(\left\|A_{\xi}\right\|^{2}+n\right) H^{\prime}-\left(n+n \alpha^{2}\right) x .
\end{aligned}
$$

In particular, if $M$ has constant mean curvature $\alpha$ in $H_{1}{ }^{n+1}$, then $D^{\prime} H^{\prime}=0$. Thus (6.13) reduces to

$$
\Delta H=-\left(\left\|A_{\xi}\right\|^{2}+n\right) H^{\prime}-\left(n+n \alpha^{2}\right) x .
$$

On the other hand, since the second fundamental form $h$ of $M$ in $E_{2}{ }^{n+2}$ is given by

$$
h(X, Y)=-\left\langle A_{\xi} X, Y\right\rangle \xi+\langle X, Y\rangle x,
$$

equation (2.7) of Gauss gives 


$$
\tau=\left\|A_{\xi}\right\|^{2}-n^{2} \alpha^{2}-n(n-1),
$$

where $\tau$ is the scalar curvature of $M$. Combining (6.14) with Lemma 1, we find

$$
\Delta H+\left(\left\|A_{\xi}\right\|^{2}+n\right) H+\left(n \alpha^{2}-\left\|A_{\xi}\right\|^{2}\right) x=0 .
$$

If the scalar curvature $\tau$ of $M$ is also constant, then (6.16) shows that

$$
b=\left\|A_{\xi}\right\|^{2}+n \text { and } c=n \alpha^{2}-\left\|A_{\xi}\right\|^{2}
$$

are constant. Therefore, Lemmas 4 and 7 imply that $M$ is of 1-type. Therefore, $x=x_{0}+x_{p}$ for some integer $p \geqq 1$. Combining this with Lemma 4 , we obtain

$$
n H=\lambda_{p}\left(x_{0}-x\right) \text {. }
$$

On the other hand, (6.17) and Lemma 5 give

$$
\left(n \alpha^{2}-\left\|A_{\xi}\right\|^{2}\right) \int_{M} x d V=0 .
$$

If $n \alpha^{2}=\left\|A_{\xi}\right\|^{2}$, then $\Delta H=-\left(n+\left\|A_{\xi}\right\|^{2}\right) H$ which is impossible. Therefore, we find $x_{0}=0$. Thus, (6.19) gives $n H=-\lambda_{p} x$. Since $x$ and $\xi$ are orthonormal, Lemma 1 implies that this case is also impossible.

(Q.E.D)

A space-like hypersurface in $H_{1}{ }^{n+1}$ (or in $H^{n+1}$ ) is said to be isoparametric if the Weingarten map $A_{\xi}$ has constant eigenvalues. Since a space-like isoparametric hypersurface in $H_{1}{ }^{n+1}$ (or in $H^{n+1}$ ) has constant mean curvature and constant scalar curvature, Theorem 2 implies immediately the following.

COROLLARY 3. There exist no compact space-like isoparametric hypersurfaces in the anti-de Sitter space-time.

\section{$\S 7$. Hypersurfaces in Hyperbolic Space.}

In this section, we apply our previous results to give the following classification theorem in hyperbolic space.

THEOREM 3. The only compact hypersurfaces with constant mean curvature and constant scalar curvature in the hyperbolic space $H^{n+1}$ are small hyperspheres of $H^{n+1}$.

Proof. We regard the hyperbolic space $H^{n+1}$ as a space-like hypersurface of the Lorentz-Minkowski world $E_{1}{ }^{n+2}$ defined by

$$
H^{n+1}=\left\{x \in E_{1}{ }^{n+2} \mid\langle x, x\rangle=-1 \text { and } t>0\right\} .
$$

Let $M$ be a compact hypersurface of $H^{n+1}$ with mean curvature vector $H^{\prime}$. Let $\xi$ be a unit vector field in the direction of $H^{\prime}$. We put $H^{\prime}=\alpha \xi$. Then, by an argument similar to that given in section 6 , we may obtain 


$$
\begin{aligned}
\Delta H= & \Delta^{D^{\prime}} H^{\prime}+2 \operatorname{tr} A_{D^{\prime} H^{\prime}}-\frac{n}{2} \operatorname{grad}\left\langle H^{\prime}, H^{\prime}\right\rangle \\
& +\left(\left\|A_{\xi}\right\|^{2}-n\right) H^{\prime}+\left(n \alpha^{2}-n\right) x .
\end{aligned}
$$

If $M$ has constant mean curvature, (7.2) reduces to

$$
\Delta H=\left(\left\|A_{\xi}\right\|^{2}-n\right) H^{\prime}+\left(n \alpha^{2}-n\right) x .
$$

On the other hand, we have

$$
\begin{gathered}
H=H^{\prime}+x, \\
\tau=n^{2} \alpha^{2}-\left\|A_{\xi}\right\|^{2}-n(n-1) .
\end{gathered}
$$

Thus, we obtain

$$
\Delta H+b H+c x=0,
$$

where $b=n-\left\|A_{\xi}\right\|^{2}$ and $c=\left\|A_{\xi}\right\|^{2}-n \alpha^{2}$. If $M$ has constant scalar curvature $\tau$, too, then (7.5) shows that $b$ and $c$ are constants. Thus, by applying Lemma $\overline{5}$, we obtain

$$
c x_{0} \int_{M} d V=c \int_{M} x d V=0 .
$$

Since $M$ lies in $H^{n+1}, t>0$. Therefore the center of mass cannot be the origin of $E_{1}{ }^{n+2}$. Thus, $c=\left\|A_{\xi}\right\|^{2}-n \alpha^{2}=0$. This implies that $M$ is totally umbilical in $H^{n+1}$. Therefore, $M$ is a small hypersphere of $H^{n+1}$ (cf. [1, p. 129].) (Q.E. D.)

Remark 4. It is well-known that a small hypersphere of $H^{n+1}$ has constant mean curvature and constant scalar curvature.

COROLLARY 4. The only compact isoparametric hypersurfaces of the hyperbolic space $H^{n+1}$ are small hyperspheres.

\section{$\S 8$. Hypersurfaces in de Sitter World.}

In this section, we study 2-type hypersurfaces in the de Sitter world.

THEOREM 4. Let $M$ be a compact space-like hypersurface in the de Sitter space-time $S_{1}{ }^{n+1} \subset E_{1}{ }^{n+2}$. Then $M$ has nonzero constant mean curvature and constant scalar curvature in $S_{1}{ }^{n+1}$ if and only if $M$ is mass-symmetric and of 2-type in the Lorentz-Minkowski world $E_{1}{ }^{n+2}$.

Proof. We recall that the de Sitter space-time $S_{1}{ }^{n+1}$ is a hypersurface of $E_{1}{ }^{n+2}$ defined by

$$
S_{1}{ }^{n+1}=\left\{x \in E_{1}{ }^{n+2} \mid\langle x, x\rangle=1\right\} .
$$


Let $H^{\prime}$ be the mean curvature vector of the compact, space-like hypersurface $M$ in $S_{1}{ }^{n+1}$. Then either $H^{\prime}=0$ or $H^{\prime}$ is time-like. Let $\xi$ be the time-like unit vector in $S_{1}{ }^{n+1}$ normal to $M$. We put $H^{\prime}=\alpha \xi$. Then we have

$$
H=H^{\prime}-x, \quad \operatorname{tr} A_{\xi}=-n \alpha .
$$

By applying an argument similar to that given in section 6 , we may obtain

$$
\begin{aligned}
\Delta H= & \Delta^{D^{\prime}} H^{\prime}+2 \operatorname{tr} A_{D^{\prime} H^{\prime}}+\frac{n}{2} \operatorname{grad}\left\langle H^{\prime}, H^{\prime}\right\rangle \\
& +\left(n-\left\|A_{\xi}\right\|^{2}\right) H^{\prime}-\left(n-n \alpha^{2}\right) x .
\end{aligned}
$$

If $M$ is mass-symmetric and of 2-type in $E_{1}{ }^{n+2}$, then there exist two constants $b$ and $c$ such that (Proposition 3)

$$
\Delta H+b H+c x=0 .
$$

Combining (8.2), (8.3) and (8.4) we find

$$
\begin{aligned}
& \Delta^{D^{\prime}} H^{\prime}+2 \operatorname{tr} A_{D^{\prime} H^{\prime}}+\frac{n}{2} \operatorname{grad}\left\langle H^{\prime}, H^{\prime}\right\rangle \\
& \quad=\left(\left\|A_{\xi}\right\|^{2}-n-b\right) H^{\prime}+\left(b-c+n-n \alpha^{2}\right) x .
\end{aligned}
$$

Since $x$ is normal to $S_{1}{ }^{n+1}$ and other terms in (8.5) are tangent to $S_{1}{ }^{n+1}$, we obtain $n \alpha^{2}=b-c+n$. Thus, $M$ has constant mean curvature $\alpha$ in the de Sitter world. Therefore, (7.5) gives

$$
\left\|A_{\xi}\right\|^{2}=n+b
$$

which is constant. From equation (2.7) of Gauss, we see that the scalar curvature $\tau$ of $M$ satisfies

$$
\tau=\left\|A_{\xi}\right\|^{2}-n^{2} \alpha^{2}+n(n-1) .
$$

Therefore, $M$ has constant scalar curvature.

Conversely, if $M$ has constant mean curvature in $S_{1}{ }^{n+1}$ and constant scalar curvature, then (8.3) implies

$$
\Delta H+b H+c x=0,
$$

where $b=\left\|A_{\xi}\right\|^{2}-n$ and $c=\left\|A_{\xi}\right\|^{2}-n \alpha^{2}$ are constant. Therefore, by applying Lemma 5 and (8.8), we see that the center of mass of $M$ in $E_{1}{ }^{n+2}$ is the origin. Thus, $M \subset S_{1}{ }^{n+1}$ is mass-symmetric in $E_{1}{ }^{n+2}$. Moreover, (8.8) and Proposition 3 show that $M$ is either of 1-type or of 2-type. If $M$ is of 1-type, Lemma 4 gives $n H=-\lambda_{p} x$. Thus, Lemma 1 implies that $M$ is minimal in the de Sitter world $S_{1}{ }^{n+1}$. This is a contradiction.

(Q.E.D.)

COROLlARY 5. Let $M$ be a compact, space-like hypersurface in the de Sitter space-time $S_{1}{ }^{n+1}$. If $M$ has nonzero constant mean curvature and constant scalar 
curvature, then

$$
\begin{aligned}
& \alpha^{2}=\left(1-\frac{\lambda_{p}}{n}\right)\left(1-\frac{\lambda_{q}}{n}\right), \\
& \tau=(n-1)\left(\lambda_{p}+\lambda_{q}\right)-\lambda_{p} \lambda_{q}>0, \\
& \left\|A_{\xi}\right\|^{2}=n-\left(\lambda_{p}+\lambda_{q}\right) .
\end{aligned}
$$

This corollary follows from Lemma 7 and the proof of Theorem 4. From Lemma 6 and Theorem 4 we also have the following.

COROLlARY 6. Let $M$ be a compact, space-like, isoparametric hypersurface of the de Sitter space-time $S_{1}{ }^{n+1} \subset E_{1}{ }^{n+2}$. Then $M$ is mass-symmetric. Moreover, if $M$ is not minimal in $S_{1}{ }^{n+1}$, then $M$ is of 2-type in $E_{1}{ }^{n+2}$.

Remark 5. We can prove that if $M$ is a compact, space-like, 2-type hypersurface of $S_{1}{ }^{n+1}$, then $M$ is always mass-symmetric in $S_{1}{ }^{n+1}$.

\section{REFERENCES}

[1] B.Y. Chen, Total Mean Curvature and Submanifolds of Finite Type, Series in Pure Math., Volume 1, World Scientific, 1984.

[2] B. Y. CHEN, 2-type submanifolds and their applications, Preprint, 1984.

[3] A. Einstein, The Meaning of Relativity, 4th Ed., Princeton Univ. Press, 1953.

[4] S.W. Hawking And G.F.R. Ellis, The Large Scale Structure of Space-Time, Cambridge Univ. Press, 1973.

[5] M. A. MAGID, Isometric immersions of Lorentz space with parallel second fundamental forms, Tsukuba J. Math. 8 (1984), 31-54.

[6] J. A. Wolf, Spaces of Constant Curvature, McGraw Hill, New York, 1967.

[7] K. Yano And B. Y. Chen, Minimal submanifolds of a higher dimensional sphere, Tensor (N.S.), 22 (1971), 369-373.

Department of Mathematics

Michigan State University

East Lansing, Michigan 48824

U.S.A. 